\title{
1-Methylnicotinamide and nicotinamide: two related anti-inflammatory agents that differentially affect the functions of activated macrophages
}

\author{
Rafał Biedroñ ${ }^{1}$, Marta Ciszek ${ }^{1}$, Marianna Tokarczyk ${ }^{1}$, Małgorzata Bobek ${ }^{1}$, \\ Maria Kurnyta ${ }^{1}$, Ewa M. Słominska ${ }^{2}$, Ryszard T. Smoleński ${ }^{2}$ and Janusz Marcinkiewicz ${ }^{1}$
}

${ }^{1}$ Department of Immunology, Jagiellonian University College of Medicine, Kraków, Poland

2 Department of Biochemistry, Medical University of Gdańsk, Poland

Received: 2007.07.10, Accepted: 2007.10.25, Published online first: 2008.03.31

\begin{abstract}
Introduction: 1-Methylnicotinamide (MNA), a major metabolite of nicotinamide (NA), is known to exert anti-inflammatory effects in vivo. Treatment of inflammatory skin diseases by topical application of MNA provides certain advantages over the use of NA. However, in contrast to NA, the molecular mechanisms of the anti-inflammatory properties of MNA are not well known. In this study the influence of exogenous MNA and NA in vitro on the generation of inflammatory mediators by macrophages $(\mathrm{M} \phi)$ was investigated.

Materials and Methods: Peritoneal $\mathrm{M} \phi$ of $\mathrm{CBA} / \mathrm{J}$ mice were activated in vitro with lipopolysaccharide and incubated with MNA or NA. The effect of these compounds on biological functions of $\mathrm{M} \phi$ was measured by evaluation of the production of reactive oxygen species (ROS) by luminol-dependent chemiluminescence, cytokines and prostaglandin $\mathrm{E}_{2}\left(\mathrm{PGE}_{2}\right)$ by ELISA, and nitric oxide (NO) by the Griess method. Moreover, the expressions of inducible NO synthase and cyclooxygenase-2 were measured by Western blotting.

Results: It was shown that at non-cytotoxic concentrations, NA inhibits the production of a variety of pro-inflammatory agents, such as tumor necrosis factor $\alpha$, interleukin $6, \mathrm{NO}, \mathrm{PGE}_{2}$, and the generation of ROS. In contrast to NA, exogenous MNA inhibited only the generation of ROS, while its effect on the synthesis of other mediators was negligible.

Conclusions: These results indicate that the anti-inflammatory properties of MNA demonstrated previously in vivo do not depend on its capacity to suppress the functions of immune cells, but more likely may be related to its action on vascular endothelium. The authors suggest that the limited permeability for exogenous MNA, in contrast to that for NA, may be responsible for its lack of suppressor activity against $\mathrm{M} \phi$.
\end{abstract}

Key words: 1 -methylnicotinamide, nicotinamide, inflammation, macrophages, ROS, cytokines.

Abbreviations: MNA - 1-methylnicotinamide, NA - nicotinamide, $\mathrm{M} \phi$ - macrophages, $\mathrm{ROS}$ - reactive oxygen species, $\mathrm{PGE}_{2}$ - prostaglandin $\mathrm{E}_{2}, \mathrm{LCL}$ - luminol-dependent chemiluminescence, $\mathrm{NO}$ - nitric oxide, iNOS - inducible NO synthase, COX-2 - cyclooxygenase-2, TNF- $\alpha$ - tumor necrosis factor $\alpha$, IFN- $\gamma$ - interferon $\gamma$, IL - interleukin, LPS - lipopolysaccharide, PBS - phosphate-buffered saline solution, LDH - lactic dehydrogenase, SDS - sodium dodecyl sulfate, RT - room temperature.

Corresponding author: Janusz Marcinkiewicz, Department of Immunology, Jagiellonian University Medical College, Czysta 18, 31-121 Kraków, Poland, tel./fax: +48 12 633-94-31, e-mail: mmmarcin@cyf-kr.edu.pl

\section{INTRODUCTION}

1-Methylnicotinamide (MNA), a major metabolite of nicotinamide (NA), has been shown recently to act in vivo as a very efficient anti-inflammatory agent [9]. After topical application of MNA, a remarkable therapeutic effect was observed in such inflammatory skin diseases as rosacea and acne vulgaris as well as in skin burns and wound healing $[22,23]$. On the other hand,
MNA therapy was not effective in cases with accompanying bacterial infection, which indicates that MNA's therapeutic effectiveness is related to its anti-inflammatory, but not anti-bacterial, properties [1]. Moreover, it was demonstrated that MNA displays an anti-thrombotic potential [3]. All these observations contradict the common view that MNA is a biologically inactive metabolite of NA [19]. However, in contrast to NA, the mechanism of MNA's anti-inflammatory activ- 


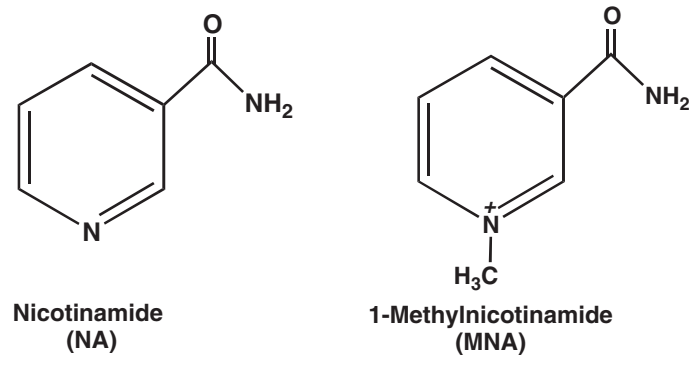

Fig. 1. Chemical structure of NA and MNA.

ity is still unclear. It remains to be examined whether MNA is able to inhibit the functions of immune cells, especially those involved in an inflammatory response (Fig. 1).

NA (niacinamide) is the amide of nicotinic acid (niacin, vitamin $\mathrm{PP}$ or $\mathrm{B}_{3}$ ) [4]. Over the years, NA has been tried in the treatment of various diseases, such as pellagra, psoriasis, diabetes, and schizophrenia [12, 21, 24]. In the last decade it has been shown that NA exerts a number of anti-inflammatory properties, e.g. inhibition of the synthesis of pro-inflammatory cytokines (tumor necrosis factor (TNF)- $\alpha$, interleukin (IL)-1, IL-6, IL-8, interferon (IFN)- $\gamma$ ), inhibition of inducible nitric oxide synthase (iNOS), modulation of free radical scavenging, and suppression of the expression of MHC class II and some adhesion molecules on immune cells [10, $11,13,14,16]$. It has been suggested that the anti-inflammatory properties of NA are related to the inhibition of NAD-dependent enzymes, such as poly(ADP-ribose) polymerase-1 $[16,20]$.

In this study we examined the anti-inflammatory potential of both MNA and NA in vitro. We chose macrophages $(M \phi)$ as representative immune inflammatory cells, potential targets for MNA and NA. In our experimental model, peritoneal murine $\mathrm{M} \phi$ activated in vitro with lipopolysaccharide (LPS) were incubated with MNA and NA and the anti-inflammatory potential of the agents was estimated by their ability to inhibit the production of selected pro- and anti-inflammatory mediators.

\section{MATERIALS AND METHODS}

\section{Animals}

Inbred $\mathrm{CBA} / \mathrm{J}$ mice (8-10 weeks old) from the Department of Immunology (Jagiellonian University Medical College, Kraków, Poland) were used. The animals were kept in a constant-climate environment with respect to the temperature $\left(22 \pm 2^{\circ} \mathrm{C}\right)$, humidity $(50 \pm 10 \%)$, and daylight cycle (light $6 \mathrm{am}-6 \mathrm{pm}$ ) and were fed standard laboratory diet with water and food ad libitum. All animal procedures conformed with the Guide for the Care and Use of Laboratory Animals published by the US National Institutes of Health (NIH
Publication No. 85-23, revised 1996) and the experimental procedures used in the present study were approved by the local Jagiellonian University Ethics Committee on Animal Experiments.

\section{Measurement of $M N A / N A$ concentration}

LPS-stimulated $\mathrm{M} \phi\left(5 \times 10^{5}\right)$ were cultured with MNA or NA used at the non-cytotoxic concentrations of 10 and $4 \mathrm{mM}$, respectively. After $24 \mathrm{~h}$ the supernatant and cells were collected separately. The cells were rapidly washed three times to remove extracellular MNA or NA and frozen until analysis. Fifty percent acetonitrile in water was used for extraction. The protein precipitate was removed by centrifugation and the supernatants were freeze dried and reconstituted with the initial mobile phase of the chromatographic system. Samples were analyzed using a liquid-chromatography mass-spectrometry system previously described elsewhere in detail [18].

\section{Macrophages}

Peritoneal mouse $\mathrm{M} \phi$ were induced by an intraperitoneal injection of $1.0 \mathrm{ml}$ of paraffin oil (Marcol 52, Exxon, USA). The cells were collected $72 \mathrm{~h}$ later by washing out the peritoneal cavity with $5 \mathrm{ml}$ of phosphate-buffered saline solution (PBS) containing $5 \mathrm{U}$ heparin/ml (Polfa, Poland). The cells were centrifuged and red blood cells were lysed by osmotic shock using distilled water. Osmolarity was restored by addition of twice-concentrated PBS. The presence of $\mathrm{M} \phi(85-90 \%)$ was judged by cytochemical demonstration of nonspecific esterase-positive mononuclear cells using $\alpha$-naphtyl acetate (Sigma, USA).

\section{Cell viability}

The effect of treatment on cell viability was assessed by measuring lactic dehydrogenase (LDH) activity in the culture supernatants with the use of the LDH Cytotoxity Detection Kit (Takara Biomedicals, Japan) and the CellTiter 96 AQueous One Solution Cell Proliferation Assay (Promega, USA) according to the manufacturers' instructions. In accordance with statistical analysis (non-linear curve fitting), the nontoxic dose ranges of NA and MNA were determined. A threshold of $90 \%$ viable cells was established for testing.

\section{Cell culture: activation of cells for the production of inflammatory mediators}

M $\phi$ stimulated with LPS (100 ng/ml) were incubated with different concentrations of MNA or NA (1-30 $\mathrm{mM})$ to test the possible anti-inflammatory activity of the agents. $\mathrm{M} \phi$ were cultured in 96-well flat-bottom cell culture plates at $1 \times 10^{5}$ cells/well in RPMI 1640 medium supplemented with $5 \%$ fetal calf serum at $37^{\circ} \mathrm{C}$ in an atmosphere of $5 \% \mathrm{CO}_{2}$. After $24 \mathrm{~h}$ of culture the cells 
and supernatants were collected and frozen at $80^{\circ} \mathrm{C}$ for further analyses.

\section{Determination of cytokines}

Cytokine concentrations in culture supernatants were measured using capture ELISA. For TNF- $\alpha$, IL-6, IL-10, and IL-12p40 determination, microtiter plates (Corning, USA) were coated overnight with rat mononuclear antibody (mAb) against a mouse cytokine (capture antibody). After blocking the plates with 3\% non-fat dried milk (for IL-10: $4 \%$ albumin), the standards and tested supernatants were added and incubated overnight. The plates were then coated with biotinylated antibodies against the same cytokine-detecting antibody for $1 \mathrm{~h}$. The ELISA was developed with horseradish peroxidase streptavidin (Vector, USA), followed by $o$-phenylenediamine and $\mathrm{H}_{2} \mathrm{O}_{2}$ (both Sigma-Aldrich, Germany) as substrates, incubated for $30 \mathrm{~min}$. The reaction was stopped with $3 \mathrm{M} \mathrm{H}_{2} \mathrm{SO}_{4}$ and the optical density of each well was measured at $492 \mathrm{~nm}$ in a 96-well plate reader. Recombinant murine cytokines were used as standard agents. $0.05 \%$ Tween 20 in phosphate buffer was used as a washing solution.

The following reagents were used for the following assays:

- IL-6: rat anti-mouse IL-6 and biotinylated rat anti-mouse IL-6 (both Pharmingen, USA) mAbs were used as capture and detecting antibodies. Recombinant mouse IL-6 (PeproTech, USA) was used as a standard. The detection limit was about $15 \mathrm{pg}$ IL-6/ml;

- IL-10: rat anti-mouse IL-10 and biotinylated rat anti-mouse IL-10 mAbs were used as capture and detecting antibodies. Recombinant mouse IL-10 was used as a standard (all reagents from Pharmingen, USA). The limit of detection was $15 \mathrm{pg}$ IL-10/ml;

- IL-12 p40: rat anti-mouse IL-12 (p40/p70) clone 15.6 (Pharmingen, USA) mAb and biotinylated rat anti-mouse IL-12(p40) clone 17.8 (Endogen, USA) mAb were used as capture and detecting antibodies. Recombinant mouse IL-12 (Genzyme, UK) was used as a standard. The detection limit was about $30 \mathrm{pg}$ IL-12 p40/ml;

- TNF- $\alpha$ : hamster anti-mouse/rat TNF- $\alpha$ and biotinylated rabbit anti-mouse/rat TNF- $\alpha$ (both Pharmingen, USA) mAbs were used as capture and detecting antibodies. Recombinant mouse TNF- $\alpha$ (Sigma, Germany) was used as a standard. The limit of detection was $30 \mathrm{pg}$ TNF- $\alpha / \mathrm{ml}$;

- prostaglandin $\mathrm{E}_{2}\left(\mathrm{PGE}_{2}\right)$ immunoassay: $\mathrm{PGE}_{2}$ concentration in supernatants was determined using a monoclonal antibody/enzyme immunoassay kit from Cayman Chemical Co. according to the manufacturer's instructions;

- nitrite determination: nitric oxide (NO), quantified by the accumulation of nitrite as a stable end-product, was determined by a microplate assay [6]. Briefly, $100-\mu \mathrm{l}$ samples were removed from the supernatants of the M $\phi$ culture and incubated with an equal volume of Griess reagent $[0.35 \%$ 4-aminophenyl sulfone (Sigma-Aldrich, Germany), 0.1\% $N$-(1-naphthyl)ethylenediamine dihydrochloride in $1 \mathrm{M} \mathrm{HCl}$ (POCh, Poland)] at room temperature for $10 \mathrm{~min}$. The absorbance at $550 \mathrm{~nm}$ was measured and nitrite concentration was calculated from a sodium nitrite standard curve.

\section{Western blot analysis of iNOS and COX-2 expression}

$\mathrm{M} \phi$ were cultured with different concentrations of MNA and NA for $24 \mathrm{~h}$ and the expressions of iNOS and cyclooxygenase-2 (COX-2) in cytosol were determined by a Western blot technique. Briefly, after incubation the cells were lysed in a lysis buffer (1\% Triton X-100, $0.1 \%$ sodium dodecyl sulfate (SDS) in PBS) containing a protease inhibitor cocktail (Sigma-Aldrich, Germany). Protein concentrations of lysates were determined using a bicinchoninic acid protein assay kit (Sigma-Aldrich, Germany). Samples containing equal amounts of total protein were mixed with gel loading buffer [0.125 M Tris, 4\% SDS, 20\% glycerol, 0.2 M DTT (dithiothreitol), $0.02 \%$ bromophenol blue] in a ratio $2: 1(\mathrm{v} / \mathrm{v})$ and boiled ( $4 \mathrm{~min})$. Then the samples $(20 \mu \mathrm{g}$ of total protein per lane) were separated on $10 \%$ SDS-polyacrylamide gels (Mighty Small II, Amersham Biosciences, USA) using a Laemmli buffer system and the proteins were transferred to nitrocellulose membranes (Bio-Rad, USA). Non-specific binding sites were blocked overnight with $3 \%$ non-fat dried milk at $4^{\circ} \mathrm{C}$ and the membranes were incubated for $2 \mathrm{~h}$ at room temperature (RT) with rabbit polyclonal antibody to iNOS (1:2000) (Stressgen, Canada) or rabbit polyclonal antibody to COX-2 (1:1000) (Cayman, USA). Bands were detected with alkaline phosphatase-conjugated secondary antibody (1 $\mathrm{h}$ at RT, 1:3000, Sigma-Aldrich, Germany) and developed with BCIP/NBT alkaline phosphatase substrate (Sigma-Aldrich, Germany). Additionally, membranes were reprobed with monoclonal anti- $\beta$-actin antibody (1 h at RT, 1:3000, Sigma-Aldrich, Germany). Prestained SDS-PAGE Standards, Low Range (Bio-Rad, USA), were used for molecular weight determinations. Protein bands were scanned and analyzed with the freeware Scion Image (Scion Corporation, USA). The data were normalized to the constitutively expressed $\beta$-actin protein.

\section{Reactive oxygen species generation: \\ luminol-dependent chemiluminescence assay}

Chemiluminescence was tested at $37^{\circ} \mathrm{C}$ in a temperature-stabilized Lucy 1 luminometer (Anthos, Austria). $\mathrm{M} \phi\left(1 \times 10^{7}\right.$ cells $\left./ \mathrm{ml}\right)$ in Hank's balanced salt solution were preincubated $\left(60 \mathrm{~min}\right.$ at $37^{\circ} \mathrm{C}$ in an atmosphere of $5 \% \mathrm{CO}_{2}$ ) on a 96-well flat-bottom black plate (Nunc, Denmark) with luminol sodium salt $(0.4 \mathrm{mg} / \mathrm{ml}$; Sigma, Germany) and with different concentrations of the agents tested $(1-30 \mathrm{mM})$. After incubation, the cells 
$(200 \mu \mathrm{l} /$ well) were quickly stimulated with opsonized zymosan $(0.4 \mathrm{mg} / \mathrm{ml})$ and photon emission over $75 \mathrm{~min}$ with 3-min intervals was measured. Each type of experiment was performed in duplicate.

\section{Statistics}

The statistical significance of differences between groups was analyzed using a factorial ANOVA (Microsoft Excel) followed by the Student's $t$-test, if appropriate. A p-value less than 0.05 was considered statistically significant.

\section{RESULTS}

\section{Distribution of exogenous MNA and NA added to the culture of LPS-stimulated M $\phi$}

To compare the cell-membrane permeability and stability of MNA and NA in vitro, the compounds were added to LPS-activated $\mathrm{M} \phi$ for $24 \mathrm{~h}$. The final intracellular (cell lysates) and extracellular (supernatants) levels of MNA and NA were estimated and compared with the initial concentrations of the compounds. As shown in Table. 1, the concentration of NA in culture medium decreased by about $20 \%$ with a concomitant increase in the intracellular level. However, NA was not metabolized to MNA by $\mathrm{M} \phi$, as the concentration of MNA in the culture was not elevated above the baseline (data not shown). The ratio of the concentration of NA measured in the culture medium to its final concentration in $\mathrm{M} \phi$ was 8.57. Under the same experimental conditions, the concentration of exogenous MNA in culture medium decreased after $24 \mathrm{~h}$ also by about $20 \%$ of the initial value. However, the ratio of the MNA concentration in the medium to the final concentration in $\mathrm{M} \phi$ was 35.8 , which is four times higher than the ratio for NA. This may indicate a limited cell-membrane permeability for MNA compared with NA. Further conversion of exogenous MNA by M $\phi$ to its metabolites 1-methyl-2-pyridone-5-carboxamide and 1-methyl-4-pyridone-5-carboxamide is unlikely to be significant since these compounds remained below the detection limit of the method (data not shown).

\section{Effect of MNA and NA}

on reactive oxygen species generation:

scavenging properties of MNA and NA

Luminol-dependent chemiluminescence (LCL) observed during the respiratory burst of $\mathrm{M} \phi$ is commonly attributed to the production of reactive oxygen species (ROS) [17]. Upon addition of opsonized zymosan, macrophage LCL increased light emission 30 times over baseline. When either MNA or NA was added to the reaction mixture, a dose dependent decrease in LCL was observed for both reagents (Fig. 2). NA was more effective than MNA. However, preincubation of the cells with either MNA or NA did not affect LCL; therefore the generation of ROS was not inhibited (data not shown). These results therefore indicate scavenging properties of the agents.

\section{Effect of MNA and NA on the production of cytokines by LPS-stimulated M $\phi$}

Stimulation of M $\phi$ in vitro with LPS resulted in a pronounced release of pro- and anti-inflammatory cytokines. Addition of NA to cultured cells at the time of activation resulted in a dose-dependent inhibition of both pro-inflammatory cytokines (TNF, IL-6). NA at concentrations below $10 \mathrm{mM}$ did not significantly affect the production of IL-12p40 and only slightly inhibited the release of IL-10, an anti-inflammatory cytokine. MNA, in contrast to NA, did not affect the release of any of the cytokines tested (Fig. 3).

\section{Effect of MNA and NA on the production of NO by LPS- and IFN- $\gamma$-stimulated $M \phi$}

In our experimental set-up, exposure of M $\phi$ to LPS and IFN- $\gamma$ resulted in the generation of nitrite $(\sim 14$ $\mu \mathrm{M})$, the end product of $\mathrm{NO}$ oxidation. As shown in Fig. 4, NA inhibited the release of nitrite in a dose-dependent manner. The highest non-cytotoxic concentration of NA $(10 \mathrm{mM})$ almost completely abrogated the generation of nitrite (Fig. 4A) without affecting the expression of iNOS (Fig. 4B). MNA at non-cytotoxic concentrations did not affect either nitrite release or the expression of iNOS.

Table. 1. Distribution of MNA and NA added to the culture of LPS-stimulated macrophages

\begin{tabular}{cccc}
\hline \multirow{2}{*}{ Exogenous compounds $(\mu \mathrm{M})^{\mathrm{a}}$} & \multicolumn{2}{c}{ Final concentration ${ }^{\mathrm{b}}$} & \multicolumn{2}{c}{$\begin{array}{c}\text { The ratio of medium } \\
\text { to cytosol concentration }\end{array}$} \\
\cline { 2 - 4 } & medium & cytosol & ND \\
MNA (0.0) & $<0.1$ & $<0.5$ & 35.8 \\
MNA (10.000) & $7991 \pm 461$ & $223 \pm 32$ & ND \\
NA (0.0) & $9 \pm \pm 1$ & $47 \pm 3$ & 8.57 \\
\hline
\end{tabular}

${ }^{\mathrm{a}} \mathrm{MNA}$ or NA was added to the culture of LPS-stimulated $\mathrm{M} \phi$ at the concentrations indicated in brackets. ${ }^{\mathrm{b}} \mathrm{After} 24 \mathrm{~h}$, supernatant and $\mathrm{M} \phi$ were collected separately and the concentrations of extracellular (medium) and intracellular (cytosol) MNA and NA were measured as described in Materials and Methods. ND - not done. 

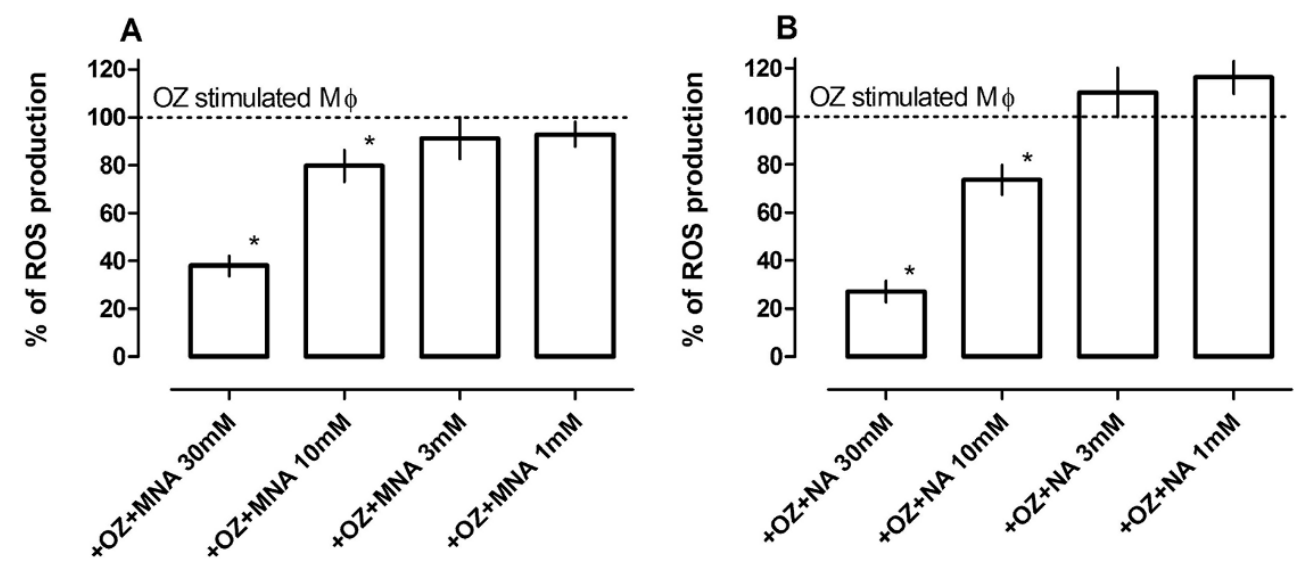

Fig. 2. Scavenging properties of MNA and NA. M $\phi\left(1 \times 10^{6}\right.$ cells/well $)$ in Hank's solution were preincubated $\left(60\right.$ min at $37^{\circ} \mathrm{C}$ in an atmosphere of $\left.5 \% \mathrm{CO}_{2}\right)$ on 96-well flat-bottom black plate with luminol sodium salt $(0.4 \mathrm{mg} / \mathrm{ml})$ and different concentrations of (A) MNA and (B) NA (1-30 mM). After incubation the cells were stimulated with opsonized zymosan $(0.4 \mathrm{mg} / \mathrm{ml})$ and photon emission was measured. Results are expressed as the percentage of ROS production by OZ-stimulated M $\phi \pm$ SEM diminished by that of non-stimulated cells. Each bar represents the mean $\left( \pm\right.$ SEM) of three individual experiments. ${ }^{*} \mathrm{p}<0.05$.
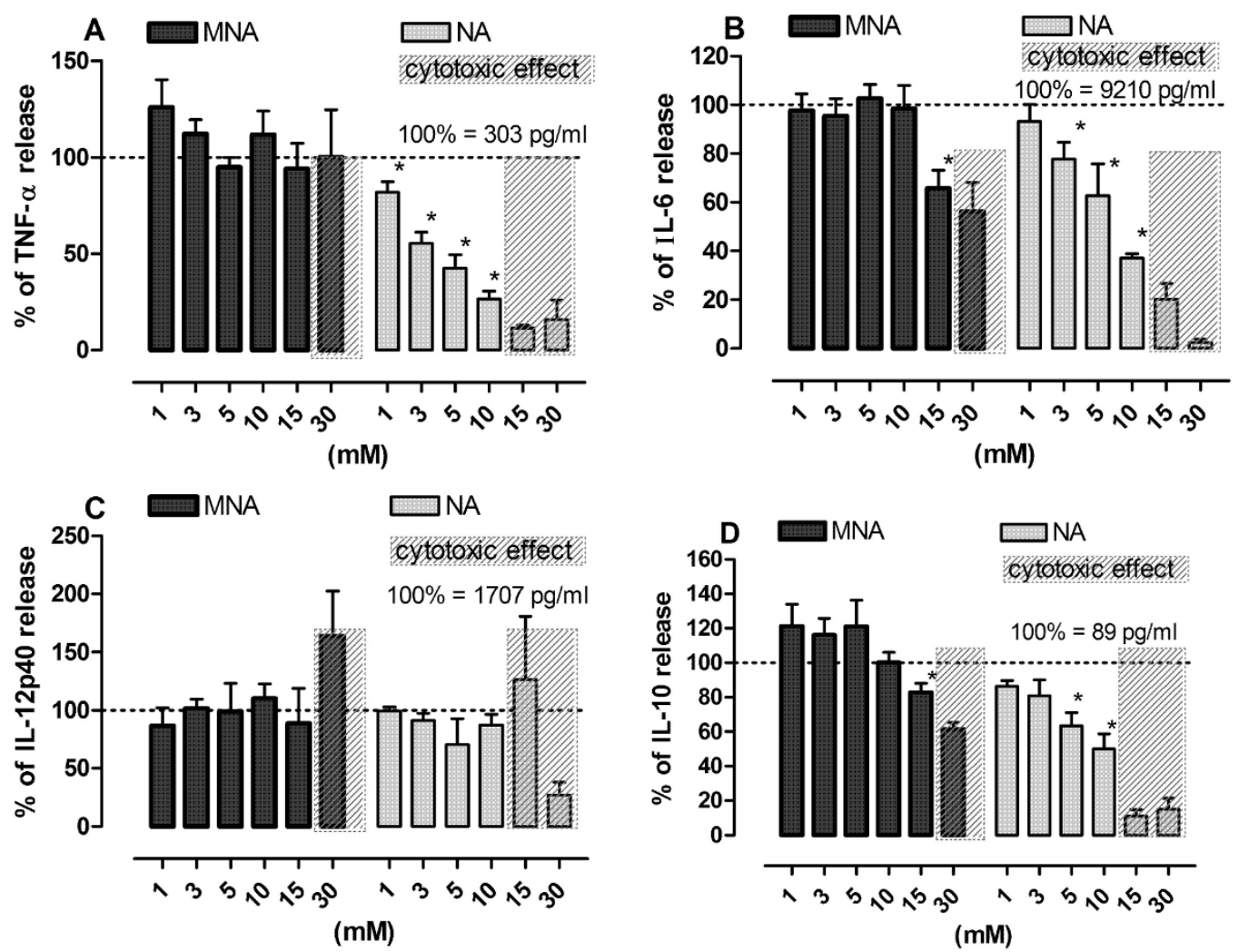

Fig. 3. Effect of MNA and NA on the production of cytokines by LPS-stimulated M $\phi$. Cells stimulated with LPS (100 ng/ml) were exposed to MNA and NA in the culture medium for $24 \mathrm{~h}$ and then the content of cytokines in the medium was measured by ELISA. A - TNF- $\alpha, \mathbf{B}-$ IL-6, C - IL-12p40, D - IL-10. Results are expressed as the percentage of the cytokine production by control M $\phi \pm$ SEM. Data were calculated 5-8 separate experiments. ${ }^{*} \mathrm{p}<0.05$.

\section{Effect of MNA and NA on prostaglandin synthesis} by LPS-stimulated M $\phi$

As shown in Fig. 5, NA, but not MNA, inhibited the production of $\mathrm{PGE}_{2}$, a major COX-2-dependent prosta- glandin produced by activated $\mathrm{M} \phi$, in a dose-dependent manner. However, Western blot analyses showed that NA did not reduce the expression of COX-2. On the other hand, a slight increase in COX-2 expression was observed in cells incubated with MNA. 

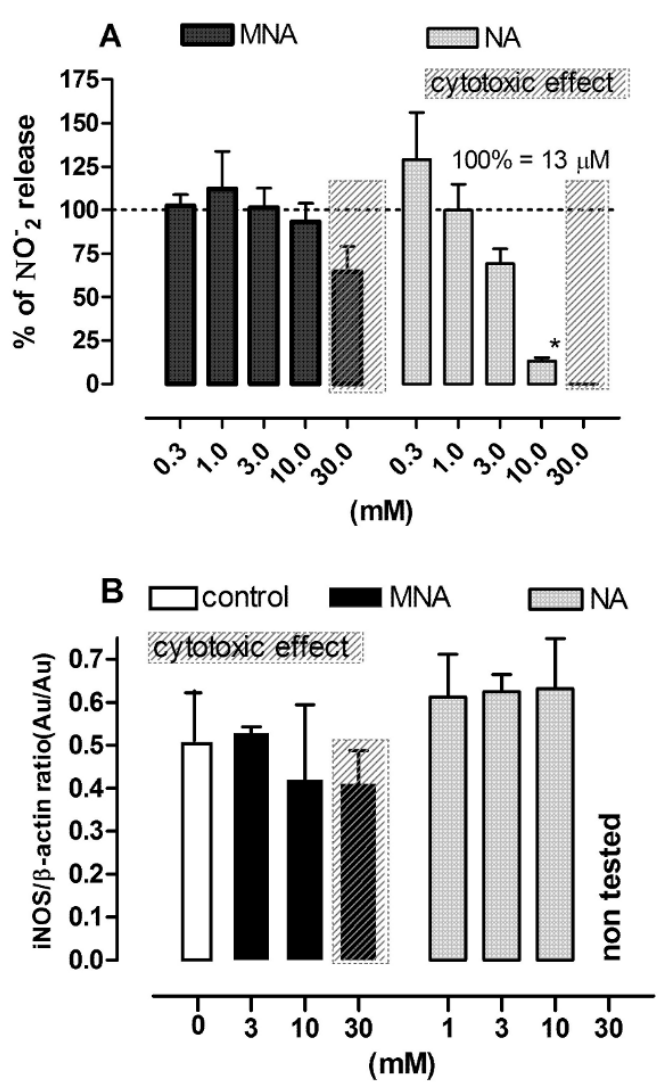

Fig. 4. Effect of MNA and NA on the production of NO and iNOS expression by LPS plus IFN- $\gamma$-stimulated $\mathrm{M} \phi$. Cells stimulated with LPS $(100 \mathrm{ng} / \mathrm{ml})$ and IFN- $\gamma(50 \mathrm{U} / \mathrm{ml})$ were exposed to MNA and NA in the culture medium for $24 \mathrm{~h}$ and then both the medium and the cells were collected for further assay. $\mathbf{A}$ - the release of nitrites $\left(\mathrm{NO}_{2}^{-}\right)$; the results are expressed as the percentage of the $\left(\mathrm{NO}_{2}^{-}\right)$production by control $\mathrm{M} \phi \pm$ SEM. Data were calculated from three separate experiments. B - the expression of iNOS; the data are normalized to constitutively expressed $\beta$-actin protein \pm SEM. Densitometric analysis of bands from two independent experiments. ${ }^{*} \mathrm{p}<0.05$.

\section{DISCUSSION}

In the present study we showed that the stimulation of peritoneal $\mathrm{M} \phi$ with LPS results in a massive secretion of a variety of inflammatory mediators, such as TNF- $\alpha$,

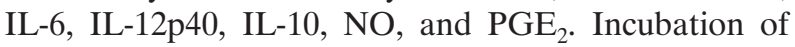
these cells with NA showed a dose-dependent reduction of the synthesis of all the mediators tested. Especially mediators with pro-inflammatory properties, such as NO, IL-6, TNF- $\alpha$, and $\mathrm{PGE}_{2}$ (the major eicosanoid produced by inflammatory phagocytic cells), were suppressed. The production of IL-10, a cytokine with anti-inflammatory potential, was inhibited to a lesser extend, which supports the anti-inflammatory properties of NA. These results are in agreement with previous observations [7, 20]. For example, it has been shown that NA is a potent inhibitor of pro-inflammatory cytokines produced by cells of the innate immune system [20]. Moreover, it has been shown that NA, but not MNA, inhibits cytokine synthesis, especially the synthesis of
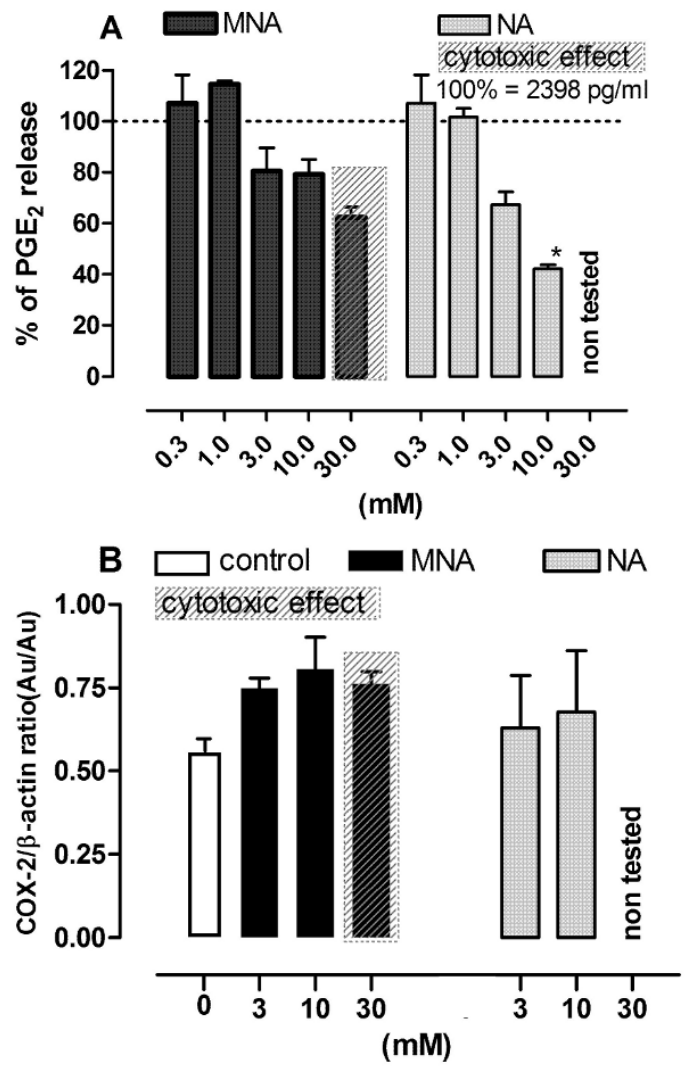

Fig. 5. Effect of MNA and NA on the production of $\mathrm{PGE}_{2}$ and COX-2 expression by LPS-stimulated macrophages. Cells stimulated with LPS (100 ng/ml) were exposed to MNA and NA in the culture medium for $24 \mathrm{~h}$ and then both the medium and the cells were collected for further assays. A - the release of $\mathrm{PGE}_{2}$; results are expressed as the percentage of the $\mathrm{PGE}_{2}$ production by control $\mathrm{M} \phi \pm$ SEM. Data were calculated from two separate experiments. B - the expression of COX-2; the data are normalized to constitutively expressed $\beta$-actin protein \pm SEM. Densitometric analysis of bands from two independent experiments. ${ }^{*} \mathrm{p}<0.05$.

TNF- $\alpha[5,8,15]$. Collectively, all these observations clearly indicate that the anti-inflammatory properties of NA are related to its ability to down-regulate functions of the cells of the immune system. However, the mechanism of anti-inflammatory activity of NA is still unclear.

MNA, in contrast to NA, as shown in this study, does not affect the functions of M $\phi$. Thus it seems to be unlikely that the anti-inflammatory properties of MNA demonstrated in vivo $[22,23]$ depend on the same mechanisms as those of NA. Examination of the therapeutic potential of MNA in cardiovascular pharmacology provides some evidence and suggestions that a major target for MNA at a site of inflammation seems to be endothelium $[3,9]$. Our investigations also support this hypothesis. We recently demonstrated that exogenous, orally administered MNA profoundly attenuates the contact hypersensitivity reaction to oxazolone in mice through endothelial $\mathrm{PGI}_{2}$-dependent mechanisms [2].

Therefore, the divergent biological activities of 
MNA and NA in vitro and their similar anti-inflammatory effect in vivo may be explained by the fact that distinct cells seem to be targets for MNA and NA. Nevertheless, the mechanisms of the different effects of exogenous MNA and NA on the functions of M $\phi$ remain to be explained. The present data show that $\mathrm{M} \phi$ are not able to metabolize exogenous NA to MNA in vitro. This clearly indicates that NA, but not its metabolites, is responsible for the observed effects. Thus it is tempting to speculate that the limited cell-membrane permeability for MNA compared with that for NA may be responsible for the lack of effect of MNA on the synthesis of inflammatory mediators by activated $\mathrm{M} \phi$. The results of the LCL experiments also support the thesis that MNA acts in the extracellular space, as it does not inhibit ROS generation but acts as a scavenging agent. On the other hand, it was found previously that MNA, but not NA, can be bound to glycosaminoglycans located on the surface of vascular endothelium cells without effective penetration of the tissue, thus increasing its local concentration on the cell surface [9].

All these observations, the lack of effect of MNA on the synthesis of inflammatory mediators in vitro together with the remarkable anti-inflammatory properties of MNA in vivo as demonstrated in the experimental contact hypersensitivity reaction in mice [2] and in the treatment of contact dermatitis in humans $[22,23]$ suggest that endothelial cells, but not immune cells, are the target for MNA. Thus in our opinion, MNA can be regarded as an effective anti-inflammatory agent, the action of which does not impair the immunity of patients.

Acknowledgment: This work was supported by the Polish Ministry of Science and Higher Education (grant no. PBZ-KBN-101/T09/2003). Our thanks are due to Prof. Jerzy Gębicki for his encouragement to carry out this study and Dr. Jan Adamus for providing us with MNA.

\section{REFERENCES}

1. Adamiec M., Adamus J., Ciebiada I., Denys A. and Gebicki J. (2006): Search for drugs of the combined anti-inflammatory and anti-bacterial properties: 1-methyl-N'-(hydroxymethyl) nicotinamide. Pharmacol. Rep., 58, 246-249.

2. Bryniarski K., Biedron R., Jakubowski A., Chłopicki S. and Marcinkiewicz J. (2008): Anti-inflammatory effect of 1-methylnicotinamide in contact hypersensitivity to oxazolone in mice; involvement of prostacyclin. Eur. J. Pharmacol., 578, 332-338.

3. Chlopicki S., Swies J., Mogielnicki A., Buczko W., Bartus M., Lomnicka M., Adamus J. and Gebicki J. (2007): 1-Methylnicotinamide (MNA), a primary metabolite of nicotinamide, exerts antithrombotic activity mediated by COX-2/PGI 2 pathway. Br. J. Pharmacol., 152, 230-239.

4. Creeke P. I. and Seal A. J. (2005): Quantitation of the niacin metabolites 1-methylnicotinamide and 1-methyl-2-pyridone-5-carboxamide in random spot urine samples, by ion-pairing reverse-phase HPLC with UV detection, and the implications for the use of spot urine samples in the assessment of niacin status. J. Chromatogr. B Analyt. Technol. Biomed. Life Sci., 817, 247-253.
5. Daniel J., Marechal Y., Van Gool F., Andris F. and Leo O. (2007): Nicotinamide inhibits B lymphocyte activation by disrupting MAPK signal transduction. Biochem. Pharmacol., 73, 831-842.

6. Ding A. H., Nathan C. F. and Stuehr D. J. (1988): Release of reactive nitrogen intermediates from mouse peritoneal macrophages: comparison of activating cytokines and evidence for independent production. J. Immunol., 141, 2407-2412.

7. Eizirik D. L., Sandler S., Welsh N., Bendtzen K. and Hellerstrom C. (1994): Nicotinamide decreases nitric oxide production and partially protects human pancreatic islets against the suppressive effects of combinations of cytokines. Autoimmunity, 19, 193-198.

8. Fukuzawa M., Satoh J., Muto G., Muto Y., Nishimura S., Miyaguchi S., Qiang X. L. and Toyota T. (1997): Inhibitory effect of nicotinamide on in vitro and in vivo production of tumor necrosis factor-alpha. Immunol. Lett., 59, 7-11.

9. Gebicki J., Sysa-Jedrzejowska A., Adamus J., Wozniacka A., Rybak M. and Zielonka J. (2003): 1-Methylnicotinamide: a potent anti-inflammatory agent of vitamin origin. Pol. J. Pharmacol., 55, 109-112.

10. Hiromatsu Y., Sato M., Tanaka K., Ishisaka N., Kamachi J. and Nonaka K. (1993): Inhibitory effects of nicotinamide on intercellular adhesion molecule-1 expression on cultured human thyroid cells. Immunology, 80, 330-332.

11. Hiromatsu Y., Sato M., Yamada K. and Nonaka K. (1992): Inhibitory effects of nicotinamide on recombinant human interferon-gamma-induced intercellular adhesion molecule-1 (ICAM-1) and HLA-DR antigen expression on cultured human endothelial cells. Immunol. Lett., 31, 35-39.

12. Maiese K. and Chong Z. Z. (2003): Nicotinamide: necessary nutrient emerges as a novel cytoprotectant for the brain. Trends Pharmacol. Sci., 24, 228-232.

13. Otsuka A., Hanafusa T., Miyagawa J., Kono N. and Tarui S. (1991): Nicotinamide and 3-aminobenzamide reduce interferon-gamma-induced class II MHC (HLA-DR and -DP) molecule expression on cultured human endothelial cells and fibroblasts. Immunopharmacol. Immunotoxicol., 13, 263-280.

14. Papaccio G., Ammendola E. and Pisanti F. A. (1999): Nicotinamide decreases MHC class II but not MHC class I expression and increases intercellular adhesion molecule-1 structures in non-obese diabetic mouse pancreas. J. Endocrinol., 160, 389-400.

15. Pero R. W., Axelsson B., Siemann D., Chaplin D. and Dougherty G. (1999): Newly discovered anti-inflammatory properties of the benzamides and nicotinamides. Mol. Cell Biochem., 193, 119-125.

16. Sánchez-Fidalgo S., Villegas I., Martín A., Sánchez-Hidalgo M. and Alarcón de la Lastra C. (2007): PARP inhibition reduces acute colonic inflammation in rats. Eur. J. Pharmacol., 563, 216-223.

17. Schleupner C. J. and Glasgow L. A. (1978): Peritoneal macrophage activation indicated by enhanced chemiluminescence. Infect. Immun., 21, 886-895.

18. Slominska E. M., Adamski P., Lipinski M., Swierczynski J. and Smolenski R. T. (2006): Liquid chromatographic/mass spectrometric procedure for measurement of NAD catabolites in human and rat plasma and urine. Nucleosides Nucleotides Nucleic Acids, 25, 1245-1249.

19. Stanulovic M. and Chaykin S. (1971): Metabolic origins of the pyridones of N1-methylnicotinamide in man and rat. Arch. Biochem. Biophys., 145, 35-42.

20. Ungerstedt J. S., Blomback M. and Soderstrom T. (2003): 
Nicotinamide is a potent inhibitor of proinflammatory cytokines. Clin. Exp. Immunol., 131, 48-52.

21. Vague P., Picq R., Bernal M., Lassmann-Vague V. and Vialettes B. (1989): Effect of nicotinamide treatment on the residual insulin secretion in type 1 (insulin-dependent) diabetic patients. Diabetologia, 32, 316-321.

22. Wozniacka A., Szajerski P., Adamus J., Gebicki J. and Sysa-Jedrzejowska A. (2007): In search for new antipsoriatic agents: NAD topical composition. Skin Pharmacol. Physiol., 20, 37-42.
23. Wozniacka A., Wieczorkowska M., Gebicki J. and Sysa-Jedrzejowska A. (2005): Topical application of 1-methylnicotinamide in the treatment of rosacea: a pilot study. Clin. Exp. Dermatol., 30, 632-635.

24. Zackheim H. S. (1978): Topical 6-aminonicotinamide plus oral niacinamide therapy for psoriasis. Arch. Dermatol., 114, 1632-1638. 